\title{
Surgical Planning for Cochlear Implantation in Far- Advanced Otosclerosis: The Utility of OTOPLAN
}

\author{
Letter to the Editor \\ Andrea Lovato (10, Cosimo de Filippis (D) \\ Department of Neuroscience DNS, University of Padova, Audiology Unit at Treviso Hospital, Treviso, Italy
}

ORCID iDs of the authors: A.L. 0000-0003-0369-1751; C.F. 0000-0002-2491-7783.

Cite this article as: Lovato A, de Filippis C. Surgical Planning for Cochlear Implantation in Far-Advanced Otosclerosis: The Utility of OTOPLAN. Turk Arch Otorhinolaryngol 2020, 58(4): 289-90.

Corresponding Author: Andrea Lovato, andrea.lovato.3@hotmail.it Received Date: 10.10 .2020 Accepted Date: 13.11.2020

Content of this journal is licensed under a Creative Commons Attribution 4.0 International License. Available online at www.turkarchotolaryngol.net
To the Editor,

We read with interest the recent investigation by Bajin et al. (1) that reviewed the management and treatment outcome of far-advanced otosclerosis (FAO) patients. The authors concluded that cochlear implantation (CI) represented a successful back-up option in cases of stapedotomy failure, in accordance to current findings by other authors (24). Bajin et al. (1) performed CI in 13 of their FAO patients $(65 \%)$, with full electrode insertion in all cases and no serious post-operative complications or side effects during follow-up. Unfortunately, the authors gave no information about the type and the length of the arrays used for surgery. The appropriate choice of CI array length represents a relevant subject, as incomplete electrode insertion remains one of the main problem in CI for FAO (3).

Recently, we considered OTOPLAN (CAScination AG; Bern, Switzerland) computer program in pre-operative decision for $\mathrm{CI}$ in $\mathrm{FAO}$ patients (4). OTOPLAN is a new software for pre-operative planning in otosurgery developed by CAScination (Bern, Switzerland) in cooperation with MED-EL (Innsbruck, Austria) (5). The software, using conventional computed tomography imaging, creates reconstructed images that give a more accurate view of cochlear lumen. Additionally, OTOPLAN calculates an estimated length for every cochlear turns and provides a report with a suggested array length to use in every patient (5). In our case series of FAO patients, we disclosed a mean OTOPLAN-estimated cochlear duct length of $32.4 \mathrm{~mm}$ (4). Furthermore, looking at OTOPLAN reconstructed imaging, we found fibrosis located in the cochlear lumen in the middle and apical turns in two FAO subjects (4). Considering all the findings from OTOPLAN software, we decided to change surgical plans and chose a shorter electrode (24 and $28 \mathrm{~mm}$ instead of $31 \mathrm{~mm}$ ) to avoid incomplete insertion (4). This software preliminarily seemed useful for the appropriate array length choice in FAO patients and should be further investigated.

Peer-review: Externally peer-reviewed.

Author Contributions: Concept - A.L., C.F.; Supervision C.F.; Writing Manuscript - A.L.

Conflict of Interest: The authors have no conflicts of interest to declare.

Financial Disclosure: The authors declared that this study has received no financial support.

\section{References}

1. Bajin MD, Ergün O, Çınar BÇ, Sennaroğlu L. Management of far-advanced otosclerosis: Stapes surgery or cochlear implant. Turk Arch Otorhinolaryngol 2020; 58: 35-40.

2. Lovato A, Kraak J, Hensen EF, Smit CF, Giacomelli L, de Filippis C, et al. A critical look into stapedotomy learning curve: influence of patient characteristics and different criteria defining success. Ear Nose Throat J 2019: 145561319866825.

3. Vashishth A, Fulcheri A, Rossi G, Prasad SC, Caruso A, Sanna M. Cochlear implantation in otosclerosis: surgical and auditory outcomes with a brief on facial nerve stimulation. Otol Neurotol 2017; 38: e345-53. 
4. Lovato A, Marioni G, Gamberini L, Bonora C, Genovese E, de Filippis C. OTOPLAN in cochlear implantation for far-advanced otosclerosis. Otol Neurotol. 2020; 41: e1024-e1028.

5. Lovato A, de Filippis C. Utility of OTOPLAN reconstructed images for surgical planning of cochlear implantation in a case of post-meningitis ossification. Otol Neurotol 2019; 40: e60-1.

\section{Author's Reply}

To the Editor,

We appreciate the letter to the editor about our paper and would like to give some further details about the subject.

The difficulty of full electrode insertion in far-advanced (FAO) otosclerosis cases is a well-known phenomenon that we have discussed in our paper. In order to avoid an unpleasing surprise we always carefully evaluate high resolution computed tomography (HRCT) scans preoperatively. We develop a clear idea about mastoid cavity, middle ear and intra-cochlear anatomy. It is a must to search for decreased intra-cochlear fluid or any obstruction at round window/basal turn in COS cases which may necessitate a cochleostomy/basal turn drilling or lead to an incomplete insertion (1).

OTOPLAN is a very helpful software to reconstruct HRCT images to evaluate intra-cochlear anatomy better and indeed may facilitate the preoperative analysis (2). Although HRCT is not an ideal imaging modality to reveal fibrosis and if authors suspect intra-cochlear fibrosis in middle or apical turns, magnetic resonance imaging would have been more appropriate.

We don't prefer using Med-E1 flex (soft) (Med-El GmbH; Innsbruck, Austria) $31 \mathrm{~mm}$ electrode array in cases with less than ideal anatomies due to the risk of incomplete insertion. We prefer Cochlear Nucleus CI422 (Cochlear Corp.; Sydney, Australia) with slim straight electrode array for FAO cases and revisions. Although it has a thin and soft atraumatic electrode array, its basal stiffener helps full insertion. Its elecrode array is inserted less than $25 \mathrm{~mm}$ which is concordant with the suggestions of the authors of the letter to the editor.

M. Demir Bajin

Department of Otorhinolaryngology-Head and Neck Surgery, Hacettepe University School of Medicine, Ankara, Turkey

Corresponding Author: M. Demir Bajin, munirdemirbajin@gmail. com

ORCID iD of the author: M.D.B. 0000-0003-1088-4367

\section{References}

1. Dumas AR, Schwalje AT, Franco-Vidal V, Bebear JP, Darrouzet $\mathrm{V}$, Bonnard D. Cochlear implantation in far-advanced otosclerosis: hearing results and complications. Acta Otorhinolaryngol Ital 2018; 38: 445-52.

2. Lovato A, Marioni G, Gamberini L, Bonora C, Genovese E, de Filippis C. OTOPLAN in Cochlear Implantation for Far-advanced Otosclerosis. Otol Neurotol. 2020; 41: e1024-e1028. 